\title{
Inhomogeneous Partition Regularity
}

\author{
Imre Leader \\ Centre for Mathematical Sciences \\ Wilberforce Road \\ Cambridge CB3 0WB, U.K.
}

I. Leader@dpmms. cam.ac.uk

\author{
Paul A. Russell \\ Churchill College \\ Cambridge CB3 0DS, U.K. \\ P.A.Russell@dpmms.cam.ac.uk
}

Submitted: Jun 29, 2018; Accepted: May 15, 2020; Published: Jun 26, 2020

(C) The authors. Released under the CC BY-ND license (International 4.0).

\begin{abstract}
We say that the system of equations $A x=b$, where $A$ is an integer matrix and $b$ is a (non-zero) integer vector, is partition regular if whenever the integers are finitely coloured there is a monochromatic vector $x$ with $A x=b$. Rado proved that the system $A x=b$ is partition regular if and only if it has a constant solution.

Byszewski and Krawczyk asked if this remains true when the integers are replaced by a general (commutative) ring $R$. Our aim in this note is to answer this question in the affirmative. The main ingredient is a new 'direct' proof of Rado's result.
\end{abstract}

Mathematics Subject Classifications: 05D10

\section{Introduction}

The study of the Ramsey properties of inhomogeneous linear equations goes back to Rado in the 1930s. Given an $m \times n$ matrix $A$, with integer entries, and a non-zero vector $b \in \mathbb{Z}^{m}$, we say that the system of equations $A x=b$ is partition regular, or partition regular over $\mathbb{Z}$, if whenever the integers are finitely coloured there is a vector $x \in \mathbb{Z}^{n}$, with all of its entries having the same colour, such that $A x=b$.

There is a trivial sufficient condition for the system $A x=b$ to be partition regular, namely that it admits a constant solution, meaning an integer vector $x$ with all of its entries the same. Rado [5] showed that, in fact, this sufficient condition is also necessary. We mention in passing that Rado also investigated the question of when there is a monochromatic vector $x$ with all entries positive such that $A x=b$, obtaining necessary and sufficient conditions in this case as well - see [5]. There is also a very large literature on the homogeneous system $A x=0$, also going back to [5] - see Graham, Rothschild and Spencer [3].

Bergelson, Deuber, Hindman and Lefmann [1] considered what happens in general (commutative) rings. So now $A$ is an $m \times n$ matrix with entries from a ring $R$, and $b$ 
is a non-zero element of $R^{m}$, and we say that the system $A x=b$ is partition regular over $R$ if, whenever $R$ is finitely coloured, there is a monochromatic vector $x \in R^{n}$ with $A x=b$. The interesting question is: when is this equivalent to the condition that there is a constant solution to $A x=b$ ? They showed that, as with $\mathbb{Z}$, this is indeed the case for a class of integral domains, namely those integral domains that are not fields but have the property that $R /(r)$ is finite for every non-zero $r \in R$. Here as usual $(r)$ denotes the ideal generated by $r$, namely $\{t r: t \in R\}$.

This work was considerably extended by Byszewski and Krawczyk [2]. They showed that the result is true for every integral domain, and also for some other cases (such as for reduced rings, meaning rings with no nilpotent elements, satisfying a certain extra condition on their prime ideals). They asked if the result holds for all rings. More generally, they also considered what happens if the vector $b$ has entries not from $R$ but from some $R$-module $M$ (and we are finitely colouring $M$ ). They showed that the condition that there is a constant solution is again equivalent, when $R$ is an integral domain and $M$ is torsion-free, and also for any $R$-module $M$ when $R$ is a Dedekind domain. Again, they asked if this condition is always equivalent (for any $\operatorname{ring} R$ and any $R$-module $M$ ) to the condition that there is a constant solution.

Our aim in this note is to answer these questions in the affirmative. The key new idea is a 'direct' approach to Rado's result. Rado himself proved his result by first proving it for a single equation (i.e. the case $m=1$ ), and then showing how one may pass from a single equation to the case of several equations. The work in [1] proceeded along the same lines, as do the results in [2] - indeed, one of the most elegant results in [2] is that if we are dealing with a single equation then the condition of partition regularity is always (for any $R$ and $M$ ) equivalent to the existence of a constant solution. The work then comes (in [1] and [2]) in the attempt to use this to build up to the case of many equations. Here, in contrast, we consider the equations 'all together'. We present a new very short proof of Rado's result that is direct (in other words, not going via the case of a single equation). And this proof, interpreted suitably, generalises to work for any ring $R$.

The plan of the paper is as follows. In Section 2 we give our proof of Rado's result, and in Section 3 we generalise to arbitrary rings (and also arbitrary modules over those rings).

\section{A new proof of Rado's result}

For completeness we restate Rado's result.

Theorem 1 (Rado [5]). Let $A$ be an $m \times n$ integer matrix and let $b \in \mathbb{Z}^{m}$ be non-zero. Then the system of equations $A x=b$ is partition regular if and only if it has a constant solution.

Proof. Let $c^{(1)}, \ldots, c^{(n)}$ be the columns of $A$, and write $s$ for their sum. Suppose that there is no constant solution: this means that $b$ does not belong to the subgroup $H$ of $\mathbb{Z}^{m}$ generated by $s$. 
Now, there must exist a group homomorphism $\theta$ from $\mathbb{Z}^{m}$ to a finite cyclic group $\mathbb{Z}_{d}$ (the integers modulo $d$ ) such that $\theta(H)=0$ and $\theta(b) \neq 0$. Indeed, the quotient $G / H$ is a product of cyclic groups (as it is finitely generated), and in this quotient the image of $b$ is non-zero, so there is a map to one of the cyclic factors that does not kill $b$. If this cyclic factor is finite we are done, while if it is infinite we compose with the projection from $\mathbb{Z}$ to $\mathbb{Z}_{d}$ for a suitable $d$.

Define a colouring of the integers with $d^{n}$ colours by colouring $t \in \mathbb{Z}$ with the $n$-tuple $\left(\theta\left(c^{(1)} t\right), \ldots, \theta\left(c^{(n)} t\right)\right)$. Suppose that for this colouring we have a monochromatic vector $x$ with $A x=b$. We have that $\theta\left(\sum c^{(i)} x_{i}\right)=\theta(b)$. Since $\theta(s)=0$, we have $\theta\left(\sum c^{(i)} x_{1}\right)=0$, and so combining these we have $\sum\left(\theta\left(c^{(i)} x_{i}\right)-\theta\left(c^{(i)} x_{1}\right)\right)=\theta(b)$. But this is a contradiction, as each term in the sum on the left-hand side is zero by our choice of colouring.

The reader familiar with the result of Straus [6] on colourings of abelian groups will notice a similarity with the product colouring above (although in [6] there is no $\theta$ to worry about). Indeed, in Byszewski and Krawczyk [2] it is Straus's result that is applied to prove their result for a single equation. The main difference is that we are working in $\mathbb{Z}^{m}$ instead of $\mathbb{Z}$. In the next section, it will also be important that above we did not 'reduce to the case when the column sum is zero', because there is (in general) no ring homomorphism that does this: this is why we work with the group homomorphism $\theta$ directly. In contrast, if one is dealing with a single equation then (as shown in [2]) one may first pass to the case when the column sum is zero, and then apply Straus's result itself.

\section{The result for general rings}

We now turn to our main result.

Theorem 2. Let $A$ be an $m \times n$ matrix with entries in a ring $R$, and let $b \in R^{m}$ be non-zero. Then the system of equations $A x=b$ is partition regular over $R$ if and only if it has a constant solution.

Proof. As before, let $c^{(1)}, \ldots, c^{(n)}$ be the columns of $A$, and write $s$ for their sum. Suppose that there is no constant solution: this means that $b$ does not belong to the subgroup $H$ of $R^{m}$ consisting of all $r s, r \in R$.

We claim that there is a group homomorphism $\theta$ from $R^{m}$ to the circle $\mathbb{T}$ such that $\theta(H)=0$ and $\theta(b) \neq 0$. This is a standard piece of group theory: let us choose a subgroup $K$ of $R^{m}$ that contains $H$ and is maximal subject to not containing the element $b$. Then the quotient map $\theta$ from $G$ to $G / K$ has $\theta(b)$ non-zero and also every non-trivial subgroup of $G / K$ must contain $\theta(b)$ (by the maximality of $K$ ). It follows from this (see e.g. Kaplansky [4]) that $G / K$ is either a cyclic group of prime-power order or else the group $\mathbb{Z}_{p^{\infty}}$ of all $p^{k}$-th roots of unity for any $k$ (for some fixed prime $p$ ). In each case this is a subgroup of $\mathbb{T}$.

Now define a colouring of $R$ with $d^{n}$ colours, where $d$ is a large positive integer, by colouring $t \in R$ with the $n$-tuple $\left(f\left(\theta\left(c^{(1)} t\right)\right), \ldots, f\left(\theta\left(c^{(n)} t\right)\right)\right)$, where $f$ is the map 
that sends the interval of the circle with arguments in $[2 \pi j / d, 2 \pi(j+1) / d)$ to $j$, each $0 \leqslant j \leqslant d-1$. Suppose that for this colouring we have a monochromatic vector $x$ with $A x=b$. We have that $\theta\left(\sum c^{(i)} x_{i}\right)=\theta(b)$. Since $\theta\left(s x_{1}\right)=0$ (as $s x_{1} \in H$ ), we have $\theta\left(\sum c^{(i)} x_{1}\right)=0$, and so combining these we have $\sum\left(\theta\left(c^{(i)} x_{i}\right)-\theta\left(c^{(i)} x_{1}\right)\right)=\theta(b)$. But this is a contradiction for $d$ large, as each term in the sum on the left-hand size has argument in $[0,4 \pi / d]$ by the definition of the colouring.

Finally, note that the above proof goes through verbatim (replacing the abelian group $R^{m}$ by the abelian group $M^{m}$ ) for $R$-modules.

Theorem 3. Let $M$ be a module over a ring $R$. Let $A$ be an $m \times n$ matrix with entries in $R$, and let $b \in M^{m}$ be non-zero. Then the system of equations $A x=b$ is partition regular over $M$ if and only if it has a constant solution.

\section{References}

[1] Bergelson, V., Deuber, D., Hindman, N. and Lefmann, H., Rado's theorem for commutative rings, J. Comb. Theory (A) 66 (1994), 68-92.

[2] Byszewski, J. and Krawczyk, E., Rado's theorem for rings and modules, arXiv: 1804.05341.

[3] Graham, R., Rothschild, B. and Spencer, J., Ramsey Theory, Wiley, New York, 1990.

[4] Kaplansky, I., Infinite Abelian Groups, University of Michigan Press, Ann Arbor, 1954.

[5] Rado, R., Studien zur Kombinatorik, Math. Zeit. 36 (1933), 242-280.

[6] Straus, E. G., A combinatorial theorem in group theory, Math. Comp. 29 (1975), 303-309. 